\title{
one-dimensional Ising model built on small-world networks: competing dynamics
}

\author{
Wei Liu $^{a}$ Wen-Yuan Xiong ${ }^{b}$ and Jian-Yang Zhu ${ }^{a, c *}$ \\ ${ }^{a}$ Department of Physics, Beijing Normal University, Beijing \\ 100875, China \\ ${ }^{b}$ Department of Physics, Hunan Lingling college, Yongzhou 425006, China. \\ ${ }^{c}$ CCAST (World Laboratory), Box 8730, Beijing 100080, China
}

\begin{abstract}
In this paper, we offer a competing dynamic analysis of the one-dimensional Ising model built on the small-world network (SWN). Adding-type SWNs are investigated in detail using a simplified Hamiltonian of mean-field nature, and the result of rewiring-type is given because of the similarities of these two typical networks. We study the dynamical processes with competing Glauber mechanism and Kawasaki mechanism. The Glauber-type single-spin transition mechanism with probability $p$ simulates the contact of the system with a heat bath and the Kawasaki-type dynamics with probability $1-p$ simulates an external energy flux. By studying the phase diagram obtained in the present work, we can realize some dynamical properties influenced by the small-world effect.
\end{abstract}

PACS number(s): 89.75.-k, 64.60.Ht, 64.60.Cn, 64.60.Fr

Since Watts and Strogatz proposed the small-world networks (SWN) [1], which are believed to catch the essence of many network systems in nature and society, a large number of further works have appeared (see [2-5] for review). Researchers are interested in investigating the properties of various models and processes on SWNs. Recently, Zhu and Zhu. successfully introduced the SWN effect to the critical dynamics of the spin system [7], and thus extended the investigation to the dynamic properties of spin models. In the 1960s, the dynamic behavior of the Ising model was successfully described with the Glauber [8] and Kawasaki [9] mechanisms. From then on, great progress has been achieved based on these two mechanisms. Besides, an interesting problem has been attracting much attention, i.e., the competing Glauber-type and Kawasaki-type dynamics [10-12]. In the competing mechanism, the Glauber-type dynamics is given probability $p$, while the Kawasaki-type one has probability $1-p$. Zhu et al.'s recent work [13] has studied small-world network effect in the competing dynamics on the Gaussian model. Some meaningful results has been obtained, but due to the requirement of the convergence of the integration, they were not able to get the full phase diagram. In this paper, we investigate the competing dynamics of the Ising model considering the small-world network effect, and we obtain the full phase diagram and the competing dynamic behavior. By this work, we can further understand the influence of the SWN effect and highlight the disparities between the dynamic mechanisms.

In the construction of SWN with a certain probability of introducing long-range links, we will end up having a whole set of possible realizations. Thus the theoretically correct way of treating dynamic systems built on SWN should involve three steps: First we have to make a full list of all the possible realizations and point out the probability of each one of them. Second, we treat the problem independently on each network. Finally, we give the expected value with all these results. Although being conceptually straightforward, it is very cumbersome even for the simplest one-dimensional Ising model. It has been suggested that the spin system on the SWN as a whole has mean-field-like effect on individual spins due to the long-range links [6,7]. Naturally, a simplified method of mean-field nature was presented by Zhu et al. [7] to describe the kinetic spin system built on SWN. According to this simplified method, all possible networks are deemed as a single one. Then, the effective Hamiltonian of a spin-lattice model built on such a network is defined as the expected value over all possible realizations.

In the present work we study two specific networks: In a one-dimensional loop, for example, (1) each randomly selected pair of vertices are additionally connected with probability $p^{A}$; and $(2)$ the vertices are visited one after another, and its link in the clockwise sense is left in place with probability $1-p^{R}$ and is reconnected to a randomly selected other node with probability $p^{R}$. Networks of higher dimensions can be built similarly. We shall refer to the first model as adding-type small-world network (A-SWN) and the second one as rewiring-type network (R-SWN).

We discuss the problem using the simplified method, and give the effective Hamiltonian first. For the onedimensional (1D) Ising model built on A-SWNs and R-SWNs, the effective Hamiltonian can be written as, respectively

$$
-\beta \mathcal{H}^{A}(\{\sigma\})=K \sum_{k} \sigma_{k}\left[\sigma_{k+1}+\frac{1}{2} p^{A} \sum_{j \neq k} \sigma_{j}\right],
$$

${ }^{*}$ Corresponding author; Electronic address: zhujy@bnu.edu.cn 


$$
-\beta \mathcal{H}^{R}(\{\sigma\})=K \sum_{k} \sigma_{k}\left[\left(1-p^{R}\right) \sigma_{k+1}+\frac{1}{N-1} p^{R} \sum_{j \neq k} \sigma_{j}\right],
$$

where $\beta=1 / k_{B} T$ and $K=\beta J$. The case of $K>0,(J>0)$, corresponds to the ferromagnetic system.

Various dynamic processes in critical phenomena are believed to be governed by two basic mechanisms, i.e., the Glauber-type with order parameter nonconserved and the Kawasaki-type with order parameter conserved. Their combination, namely the competing dynamics, gives

$$
\begin{gathered}
\frac{d}{d t} P(\{\sigma\}, t)=p G_{m e}+(1-p) K_{m e}, \\
\frac{d}{d t} q_{k}(t)=p Q_{k}^{G}+(1-p) Q_{k}^{K}, q_{i}(t)=\sum_{\{\sigma\}} \sigma_{i} P(\{\sigma\}, t) .
\end{gathered}
$$

This dynamic competition has also been receiving attention $[12,13]$. Here, $p G_{m e}$ (or $p Q_{k}^{G}$ ) denotes the Glauber-type mechanism with probability $p$ and $(1-p) K_{m e}\left[\operatorname{or}(1-p) Q_{k}^{K}\right]$ denotes the Kawasaki-type mechanism with probability $1-p$; they are determined, respectively, by the Glauber-type single-spin transition probability $W_{i}\left(\sigma_{i} \rightarrow \hat{\sigma}_{i}\right)[14]$ and by the Kawasaki-type pair-spin redistribution probability $W_{j l}\left(\sigma_{j} \sigma_{l} \rightarrow \hat{\sigma}_{j} \hat{\sigma}_{l}\right)$ [15].

In their original form, the Glauber-type dynamics and the Kawasaki-type dynamics both favor a lower energy state. However, the competing dynamics is usually used to describe a system in contact with a heat bath while exposed to an external energy flux. Naturally one requires a competition between one process favoring lower system energy and the other one favoring higher system energy. Usually, the Glauber-type mechanism is used to simulate the contact of the system with a heat bath and it favors a lower energy state. On the other hand the Kawasaki-type mechanism can be modified to simulate an external energy flux that drives the system towards a higher energy state. This can be achieved by switching $\beta$ to $-\beta$, or $K=\beta J=J / K_{B} T$ to $-K$, and modifying the redistribution probability accordingly. This means that the competition between the Glauber-type mechanism and the Kawasaki-type mechanism is actually a competition between ferromagnetism and antiferromagnetism. Probing the competing behavior of the 1D Ising on A-SWNs and R-SWNs is certainly a problem of interest. The equation of evolution of the local magnetization is given by

$$
\frac{d}{d t} q_{k}(t)=p Q_{k}^{G^{A, R}}+(1-p) Q_{k}^{K^{A, R}} .
$$

The first and the second term correspond, respectively, to the part of the Glauber's dynamics with probability $p$, and the part of the modified Kawasaki's dynamics (only switching $K=\beta J$ to $-K$ ) with probability $1-p$. Our calculation will focus on A-SWN, while the result of R-SWN will be given straightforwardly.

For the Glauber-type dynamics

$$
Q_{i}^{G^{A}} \equiv-q_{i}(t)+\sum_{\{\sigma\}}\left[\sum_{\hat{\sigma}_{i}} \hat{\sigma}_{i} W_{i}^{A}\left(\sigma_{i} \rightarrow \hat{\sigma}_{i}\right)\right] P(\{\sigma\}, t),
$$

and for the Kawasaki-type dynamics

$$
\begin{aligned}
Q_{k}^{K^{A}} \equiv & -2 q_{k}(t)-p^{A}(N-1) q_{k}(t)+\sum_{\{\sigma\}}\left\{\sum_{\omega= \pm 1}\left[\sum_{\hat{\sigma}_{k}, \hat{\sigma}_{k+\omega}} \hat{\sigma}_{k} W_{k, k+\omega}^{A}\left(\sigma_{k} \sigma_{k+\omega} \rightarrow \hat{\sigma}_{k} \hat{\sigma}_{k+\omega}\right)\right]\right. \\
& \left.+p^{A}\left[\sum_{l \neq k} \sum_{\hat{\sigma}_{k}, \hat{\sigma}_{l}} \hat{\sigma}_{k} W_{k l}^{A}\left(\sigma_{k} \sigma_{l} \rightarrow \hat{\sigma}_{k} \hat{\sigma}_{l}\right)\right]\right\} P(\{\sigma\}, t),
\end{aligned}
$$

where the expressions of $W_{i}\left(\sigma_{i} \rightarrow \hat{\sigma}_{i}\right)$ and $W_{j l}\left(\sigma_{j} \sigma_{l} \rightarrow \hat{\sigma}_{j} \hat{\sigma}_{l}\right)$ can be found in Ref. [7] (but differently from that paper, we should switch $\beta$ to $-\beta$ for $W_{j l}$ ). Three important combined terms in Eqs. (6) and (7) are calculated, for the 1D Ising model, to be

$$
\sum_{\hat{\sigma}_{k}} \hat{\sigma}_{k} W_{k}^{A}\left(\sigma_{k} \rightarrow \hat{\sigma}_{k}\right)=\tanh \left[K\left(\sigma_{k-1}+\sigma_{k+1}\right)+K(N-1) p^{A} \bar{\sigma}\right]
$$




$$
\begin{gathered}
\sum_{\hat{\sigma}_{k}, \hat{\sigma}_{k \pm 1}} \hat{\sigma}_{k} W_{k, k \pm 1}^{A}\left(\sigma_{k} \sigma_{k \pm 1} \rightarrow \hat{\sigma}_{k} \hat{\sigma}_{k \pm 1}\right) \\
=\frac{\sigma_{k}+\sigma_{k \pm 1}}{2}+\frac{1}{4}\left(\sigma_{k \mp 1}-\sigma_{k \pm 2}-\sigma_{k \mp 1} \sigma_{k} \sigma_{k \pm 1}+\sigma_{k} \sigma_{k \pm 1} \sigma_{k \pm 2}\right) \tanh (2 K), \\
\sum_{l(l \neq k)} \sum_{\hat{\sigma}_{k}, \hat{\sigma}_{l}} \hat{\sigma}_{k} W_{k l}^{A}\left(\sigma_{k} \sigma_{l} \rightarrow \hat{\sigma}_{k} \hat{\sigma}_{l}\right) \\
=\frac{1}{2}(N-1)\left(\sigma_{k}+M\right)+\sum_{l(l \neq k)} \frac{1}{2}\left(\sigma_{k}-\sigma_{l}\right) \tanh \left\{K\left[\sigma_{k}\left(\sigma_{k-1}+\sigma_{k+1}-\sigma_{l-1}-\sigma_{l+1}\right)\right]\right\} .
\end{gathered}
$$

Now we turn to determine the system behavior by studying the tendency of evolution of the following order parameters:

$$
M(t) \equiv \frac{1}{N} \sum_{k} q_{k}(t), M^{\prime}(t) \equiv \frac{1}{N} \sum_{k}(-1)^{k} q_{k}(t) .
$$

Obviously, a state with both vanishing $M(t)$ and $M^{\prime}(t)$ corresponds to the disordered paramagnetic phase; a state with nonvanishing $M(t)$ and vanishing $M^{\prime}(t)$ corresponds to the ferromagnetic phase; and, in an antiferromagnetic phase we will find vanishing $M(t)$ and nonvanishing $M^{\prime}(t)$. If both order parameters are nonvanishing, this phase cannot be simply identified as a ferromagnetic or paramagnetic phase, but can be tentatively named as a heterophase.

The cases of A-SWN will be discussed in detail in the following, and we will determine the tendency of system evolution under a small perturbation $M(t) \rightarrow 0$. We then give the results of the case of R-SWN.

For the 1D Ising model built on A-SWNs, the effective Hamiltonian of the system is given by Eq. (1). From Eqs. (6)-(8), we can easily obtain

$$
\begin{gathered}
M(t)=M_{0} \exp \left\{-p\left[(1-\tanh 2 K)-K(N-1) p^{A}\left(1-\frac{1}{2} \tanh ^{2} 2 K\right)\right] t\right\}, \\
M^{\prime}(t)=M_{0}^{\prime} \exp [-\{p(1+\tanh 2 K) \\
\left.\left.+(1-p)\left[2-\tanh 2 K+\frac{1}{2}(N-1) p^{A}\left(1-\frac{1}{2} \tanh 2 K-\frac{1}{4} \tanh 4 K\right)\right]\right\} t\right] .
\end{gathered}
$$

The tendency of evolution of the order parameters $M(t)$ and $M^{\prime}(t)$ can be demonstrated by Eqs. (9) and (10) when the system undergoes a small perturbation.

(1) When $p^{A}=0$, it means that no long-range link exists, and

$$
\begin{gathered}
M(t)=M_{0} \exp \left[-p\left(1-\frac{\tanh 2 K}{\tanh 2 K_{c}^{0}}\right) t\right], \\
M^{\prime}(t)=M_{0}^{\prime} \exp \left\{-\left[p\left(1+\frac{\tanh 2 K}{\tanh 2 K_{c}^{0}}\right)+(1-p)\left(2-\frac{\tanh 2 K}{\tanh 2 K_{c}^{0}}\right)\right] t\right\},
\end{gathered}
$$

where $\tanh 2 K_{c}^{0}=1$ corresponds to the critical point of the one-dimensional (1D) Ising model without the SWN effect $\left(K_{c}^{0} \rightarrow \infty, T_{c}^{0}=0\right)$. When $K<K_{c}^{0},\left(T>T_{c}^{0}=0\right)$, Eqs. (11) and (12) show that $M(t)$ and $M^{\prime}(t)$ are both approaching zero exponentially due to the fact that tanh $2 K<1$, and thus we can reach the conclusion that, by whatever amount one increases the energy flux, the system will stay in the paramagnetic phase at arbitrary finite temperature. When $K \rightarrow K_{c}^{0}, M^{\prime}(t) \rightarrow 0$, and

$$
M(t)=M_{0} \exp \left[-p \frac{t}{\tau}\right], \tau=\frac{1}{1-\tanh (2 K) / \tanh \left(2 K_{c}^{0}\right)} \rightarrow \infty,
$$

the critical slowing down of the order parameter $M(t)$ will appear at the critical point $K_{c}^{0}$.

(2) Now a small portion of adding-type long links are introduced to the system, the system behavior in this case can be described by Eqs. (9) and (10). Obviously, $M^{\prime}(t)$ is approaching zero exponentially at any temperature and any $p^{A}$. From Eqs. (10) and (12), we can see clearly that the rate of $M^{\prime}(t)$ approaching zero is faster than that in 
the regular network. Different from $M^{\prime}(t)$, the evolution tendency of the order parameter $M(t)$ depends on both $K$ and $p^{A}$. We can obtained the critical point by the following equation:

$$
\tanh 2 K_{c}^{A}+(N-1) p^{A} K_{c}^{A}\left(1-\frac{1}{2} \tanh ^{2} 2 K_{c}^{A}\right)=1 .
$$

To give an example, if we suppose $p^{A}=1 / N$, we can get $\left.\tanh 2 K_{c}^{A}\right|_{p^{A} \sim 1 / N}=0.6809$, or $\left.K_{c}^{A}\right|_{p^{A} \sim 1 / N}=0.154$. Relative to $K_{c}^{A}$, when $t \rightarrow \infty, M(t) \rightarrow 0$ for $K<K_{c}^{A}, M(t) \neq 0$ for $K>K_{c}^{A}$, and $M(t)$ experiences critical slowing down for $K \rightarrow K_{c}^{A}$. So, combining $M(t)$ and $M^{\prime}(t)$ we can conclude that: (2.a) For the case of $K<K_{c}^{A}$, the system stays in the paramagnetic phase; (2.b) For the case of $K \rightarrow K_{c}^{A}$, the system shows the critical slowing down; and (2.c) For the case of $K>K_{c}^{A}$, the system stays in the ferromagnetic phase.

However, when $p=0$, we cannot identify the system simply as ferromagnetic or paramagnetic. Because in this case, it depends on the initial state. If $M_{0} \neq 0$, the system will stay in ferromagnetic, otherwise the system will be paramagnetic.

The phase diagram is shown in Fig. 1(a).

For the 1D Ising model built on R-SWNs, the effective Hamiltonian of the system is given by Eq. (2). With analogous calculation, one can get the equation of the critical point $K_{c}^{R}$

$$
\tanh \left[2 K_{c}^{R}\left(1-p^{R}\right)\right]+2 K_{c}^{R} p^{R}\left\{1-\frac{1}{2} \tanh ^{2}\left[2 K_{c}^{R}\left(1-p^{R}\right)\right]\right\}=1,
$$

and the time-evolution of the orders parameters $M(t)$ and $M^{\prime}(t)$

$$
\begin{aligned}
M(t)= & M_{0} \exp \left\{-p\left(1-\tanh \left[2 K\left(1-p^{R}\right)\right]-2 K p^{R}\left\{1-\frac{1}{2} \tanh ^{2}\left[2 K\left(1-p_{R}\right)\right]\right\}\right) t\right\}, \\
M^{\prime}(t)= & M_{0}^{\prime} \exp \left[-\left\{p\left(1+\tanh \left[2 K\left(1-p_{R}\right)\right]\right)+(1-p)\left[\left(1-p^{R}\right)\left\{2-\tanh \left[2 K\left(1-p_{R}\right)\right]\right\}\right.\right.\right. \\
& \left.\left.\left.+\frac{1}{2} p^{R}\left\{1-\frac{1}{2} \tanh \left[2 K\left(1-p_{R}\right)\right]-\frac{1}{4} \tanh \left[4 K\left(1-p_{R}\right)\right]\right\}\right]\right\} t\right] .
\end{aligned}
$$

(1) When no rewiring long link exists, i.e., $p^{R}=0$, the evolution is the same as the case of $p^{A}=0$.

(2) When a small portion of rewiring-type long links is introduced to the system, $M^{\prime}(t)$ is approaching zero exponentially at any temperature and any $p^{R}$. The decay rate of $M^{\prime}(t)$ is also faster then before. Different from $M^{\prime}(t)$, the evolution tendency of the order parameter $M(t)$ depends on both $K$ and $p^{R}$. The calculation of the critical point is similar to the A-SWN one. When $p^{R}=0.1$, we can get the critical point, $\left.\tanh \left[2 K_{c}^{R}\left(1-p^{R}\right)\right]\right|_{p^{R}=0.1}=0.90222$, or $\left.K_{c}^{R}\right|_{p^{R}=0.1}=0.82446$. Relative to $K_{c}^{R}$, when $t \rightarrow \infty, M(t) \rightarrow 0$ for $K<K_{c}^{R}, M(t) \neq 0$ for $K>K_{c}^{R}$, and $M(t)$ shows critical slowing down for $K \rightarrow K_{c}^{R}$. Combining $M(t)$ and $M^{\prime}(t)$ we can conclude that: (2.a) For the case of $K<K_{c}^{R}$, the system stays in the paramagnetic phase; (2.b) For the case of $K \rightarrow K_{c}^{R}$, the system shows the critical slowing down; and (2.c) For the case of $K>K_{c}^{R}$, the system stays in the ferromagnetic phase.

The phase diagram is shown in Fig. 1(b).

In this paper, we analytically study the dynamic properties of the 1D Ising model built on small-world networks. Two typical SWNs are investigated, the adding type and rewiring type. As is generally known, the 1D Ising model on the regular lattice does not show continuous phase transition at any nonzero temperature. However, if the SWN effect is introduced, critical phenomena appear in the 1D Ising model. With competing dynamics, as long as $p \neq 0$, the phase diagrams are separated into two regions. Below the critical temperature, the system will get into the ferromagnetic phase, while above the temperature, the system will get into the paramagnetic phase. The critical temperature is independent of the competing probability $p$. Different from the 2D Ising model [10] and the Gaussian model [13], the 1D Ising model built on SWNs does not show antiferromagentic phase at any temperature and any competing probability $p$.

As we have seen above, the Ising model shows critical phenomena on both A-SWNs and R-SWNs. This is because random links introduce long-range interactions. It is reasonable that the system will exhibit long-range order at finite temperature. Furthermore, the more extra links, the higher the critical temperature. Thus on R-SWNs the Ising model has a maximum critical temperature for there is a maximum number of the random links (it is also expected that the simplified method with an effective Hamiltonian will fail when most regular links are rewired). However, long-range interactions do not lead to antiferromagnetic order at any competition probability, but instead, the long range links make any antiferromagnetic order decay faster. This is because the long-range interaction here is random, while antiferromagnet needs ordered long interactions. 
The work was supported by the National Natural Science Foundation of China (No. 10375008), and the National Basic Research Program of China (2003CB716302).

[1] D. J. Watts and S. H. Strogatz, Nature 393, 440 (1998).

[2] R. Albert and A.-L. Barabasi, Rev. Mod. Phys.74 47 (2002).

[3] S. H. Strogatz, Nature (London) 410, 268 (2001).

[4] S. N. Dorogovtsev and J. F. F. Mendes, Adv. Phys. 51, 1079 (2002).

[5] M. E. J. Newman, SIAM Rev. 45167 (2003).

[6] A. Barrat and M. Weigt, Eur. Phys. J. B 13547 (2000); H. Hong, B. J. Kim and M. Y. Choi, Phys. Rev. E 66, 018101 (2002); C. P. Herrero, Phys. Rev. E 65, 066110 (2002); B. J. Kim et al., Phys. Rev. E 64, 056135 (2001).

[7] J.-Y. Zhu and H. Zhu, Phys. Rev. E 67, 026125 (2003).

[8] R. J. Glauber, J. Math. Phys. 4, 294 (1963).

[9] K. Kawasaki, Phys. Rev. 145, 224 (1966).

[10] T. Tome, M.J. de Oliveira, Phys. Rev. A 40, 6643 (1989).

[11] B.C.S. Grandi and W. Figueiredo, Phys. Rev. E 53, 5484 (1996); 54, 4722 (1996); 56, 5240 (1996); Phys. Rev. B 50,12595 (1994); Mod. Phys. Lett. B 10, 945 (1996); M. Yuqiang, L. Jiwen, Phys. Lett. A 238, 159 (1998); J.R.S. Leao, B.C.S. Grandi, W. Figueiredo, Phys. Rev. E 60, 5367 (1999); S. Bekhechi et al., Phys. Rev. E 64, 016134 (2001); M. Godoy, W. Figueiredo, Phys. Rev. E 65, 026111 (2002).

[12] H. Zhu, J.-Y. Zhu and Y. Zhou, Phys. Rev. E 66, 036106 (2002).

[13] J.-Y. Zhu, W. Liu and H. Zhu, Eur. Phys. J. B 36, 545(2003)

[14] J.-Y. Zhu and Z. R. Yang, Phys. Rev. E 59, 1551 (1999); 61, 210 (2000); E 61, 6219 (2000).

[15] H. Zhu and J.-Y. Zhu, Phys. Rev. E 66, 017102 (2002).

\section{Caption of figures}

Fig.1. The phase diagrams of $1 D$ Ising model built on SWN with competing dynamics, Glauber-type with probability $p$, and Kawasaki-type with probability $(1-p)$. (a) On the A-SWN structure: $p^{A} \sim 1 / N$; (b) On the R-SWN structure: $p^{R}=0.1$. In which, $\tanh 2 K_{c}^{0}=1$ corresponding to the critical point of the $1 D$ Ising model without the SWN effect $\left(K_{c}^{0} \rightarrow \infty, T_{c}^{0}=0\right)$. 
(a) $A-S W N: p^{A} \sim 1 / N$

(b) $R-S W N: p^{R}=0.1$
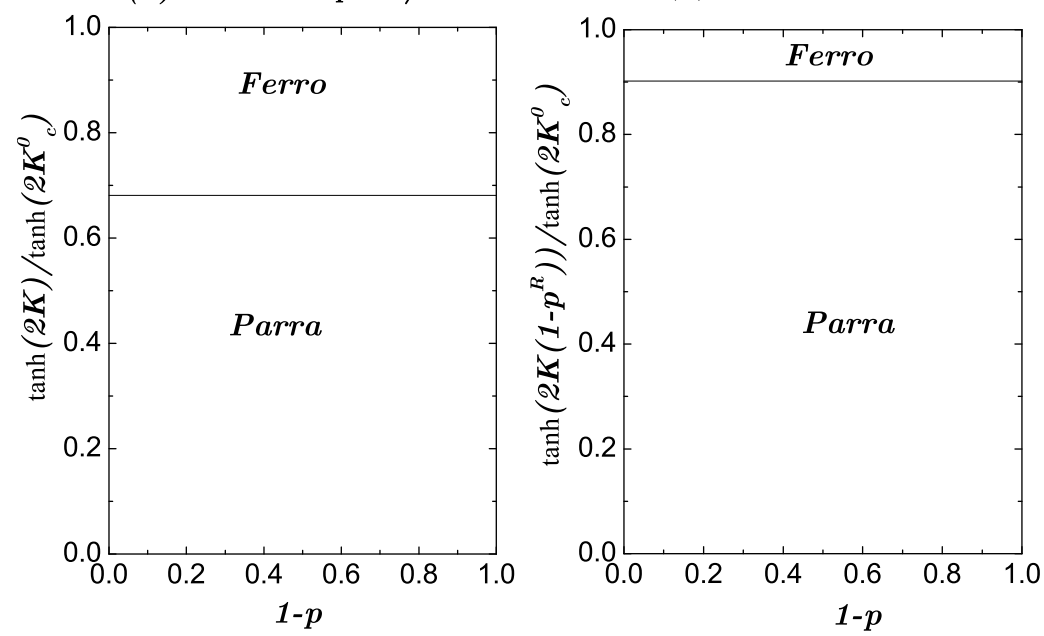